\title{
Intervenções na atividade de leitura em textos digitais: um sistema de coordenação para promover feedbacks em grupo
}

\author{
Ramon Rosa Maia Vieira Junior, Eliseo Berni Reategui \\ Programa de Pós-Graduação em Informática na Educação (PPGIE) \\ Universidade Federal do Rio Grande do Sul (UFRGS) \\ ramon.maia@ufrgs.br, eliseoreategui@gmail.com
}

\begin{abstract}
Resumo. $O$ objetivo desse trabalho é avaliar um sistema de coordenação de intervenções na leitura em textos digitais por meio de análise dos impactos nos acervos de autorias e na ocorrência de feedbacks. O sistema de coordenação foi implementado no HiperDidático, o qual foi desenvolvido para atender o fluxo de atividades colaborativas formalizado pela Arquitetura Pedagógica para Leitura de Textos Digitais (APLT). Os procedimentos metodológicos foram divididos em 3 etapas: (i) aplicação da Arquitetura Pedagógica para Leitura de Textos Digitais em uma atividade de leitura com uma turma de 15 alunos, (ii) análise da correlação entre as respostas às intervenções e os registros do acervo de certezas $e$ dúvidas dos alunos, (iii) análise da correlação entre a produção de autorias e a ocorrência de feedbacks. Os resultados evidenciaram que há uma correlação estatística pouco significativa entre as intervenções e a reconstrução do acervo, porém a segunda análise apresentou uma alta correspondência entre a produção de autorias e a ocorrência de feedbacks em um nível de significância 'altamente significativo'. Os resultados sugerem um sistema de coordenação capaz de ampliar a ocorrência de feedbacks, mesmo com baixa produção de autorias.
\end{abstract}

Palavras-chaves: coordenação, intervenção, leitura, texto digital, pergunta

\section{Interventions in Reading with Digital Texts: A Coordination System to promote Group Feedbacks}

\begin{abstract}
This paper aims to evaluate a system of coordination of interventions in reading in digital texts by means of analysis of the impacts on the authors' collections and the occurrence of feedbacks. The coordination system was implemented in HiperDidático, the groupware which was developed to support the workflow of collaborative activities formalized by Pedagogical Architecture to Reading Digital Texts (APLT). The methodological procedures were divided into 3 stages: ( $i$ ) application of the (APLT) in a reading activity with a group of 15 students, (ii) analysis of the correlation between the answers to the interventions and the records of the student's certainty and doubts, (iii) analysis of the correlation between authorships and feedbacks. The results has showed that there is a low statistical correlation between the interventions and the reconstruction of the collection, but the second analysis has showed a high correspondence between the production of authorship and the occurrence of feedbacks at a 'highly significant' level of significance. The results suggest a coordination system capable of increasing the occurrence of feedbacks, even with low authorship production.
\end{abstract}

Keywords: coordination, intervention, reading, digital text, question

\section{Introdução}

Cada vez mais os softwares têm contribuído para a ampliação do trabalho colaborativo oferecendo novas interfaces e maior interatividade em favor da aprendizagem em rede. A inovação de propostas pedagógicas colaborativas encaminha às atividades de coordenação a responsabilidade de implementar 
os protocolos de interação, ampliar as oportunidades de trocas, facilitar a cooperação e evitar os conflitos. As dinâmicas em grupo presenciais são geralmente coordenadas pelo professor - mediador do processo - assumindo-o como responsável pela definição dos papéis e regras, nos cumprimentos dos prazos, na gestão dos conflitos e, portanto, na especificação dos protocolos de interação.

As atividades em grupo realizadas nos espaços digitais (groupwares) estendem as características da presencialidade e do espaço físico, todavia introduzem, também, uma outra cultura de interatividade, na qual impõe novas formas de organização e de coordenação. A nova lógica imposta pelo ciberespaço - composta pelas interseções das dimensões tempo-espaço - emprega um conjunto de facilidades e dificuldades. Facilidades como a reescrita, a presencialidade virtual em múltiplos espaços, a possibilidade de manejar atividades concorrentes, a manipulação e a disponibilidade de diferentes mídias permitem ampliar as possibilidades de interação (humano-artefato e humano-humano). Em contrapartida, novos desdobramentos - inerentes ao ciberespaço - também potencializam a ocorrência de conflitos nas ações dos participantes. Entre outros exemplos, a falta de monitoramento das atividades e a baixa percepção (awareness) sobre o progresso das ações entre os pares podem promover atividades conflitantes ou repetitivas. Na medida que o número de participantes, de regras de cooperação e de artefatos gerados aumentam, a coordenação do trabalho colaborativo torna-se mais complexo.

Nesse sentido, as Arquiteturas Pedagógicas (APs) formalizam um encontro entre propostas de aprendizagem e apoio computacional em software, o que demanda do sujeito, sobretudo, regulações ativas (atividades cognitivas constituídas essencialmente por coordenações inferenciais próprias das metodologias ativas - active learning). As propostas estruturam desafios aos sujeitos por meio de atividades cooperativas, direcionando-os às situações de desequilíbrios (cognitivo) e promovendo cenários para apoiá-los (ex. intervenções e feedbacks) a superá-las. Durante esse percurso de [re]construções, os sujeitos são submetidos a diferentes atividades individuais e coletivas de escrita, leitura e entre outras ações sobre diferentes artefatos (ex. mídias), tendo em vista a construção de novas compreensões.

Este trabalho investiga o sistema de coordenação da Arquitetura Pedagógica para Leitura Digital (APLT) [Vieira Junior e Reategui, 2018]. A APLT tem por concepção apoiar a atividade de leitura em textos digitais através de um conjunto de intervenções e apoiado pelo suporte computacional - Groupware HiperDidático. O HiperDidático possui um sistema de coordenação do trabalho colaborativo com objetivo de coordenar as intervenções antes, durante e depois da leitura. As intervenções ocorrem no formato de perguntas fixas, programadas e automáticas.

A APLT possui três elementos responsáveis pelo conjunto de autorias produzidas: as respostas das perguntas, o acervo e a síntese. As respostas e o acervo compõem os elementos estruturantes da APLT. As respostas são elaboradas, essencialmente, a partir das perguntas programadas, enquanto o acervo e a síntese são construídos a partir das perguntas fixas. $\mathrm{O}$ acervo corresponde ao inventário de conhecimentos elaborados pelos leitores - composto por suas certezas temporárias (a consciência "do que se sabe") e dúvidas provisórias (a consciência "do que não se sabe"). Em conformidade com a concepção da APLT, o HiperDidático [HiperDidático, 2019] provém um ambiente de autoria, no qual se permite revisar e registrar as certezas/dúvidas em qualquer momento da leitura - antes, durante ou depois.

O conjunto das intervenções possui três principais objetivos: (1) extrair dos leitores as formalizações de certezas e dúvidas, (2) criar situações favoráveis para reflexões dos sujeitos sobre suas formalizações - respostas e acervo - reconstruindo-as progressivamente e (3) promover feedbacks aos leitores em um sistema de trocas em rede.

O objetivo deste trabalho é avaliar o sistema de coordenação de intervenções na leitura em textos digitais sob o formato de hiperdocumentos, implementado no HiperDidático, no qual foi 
desenvolvido em conformidade com as atividades formalizadas pela APLT. A pesquisa procede em uma investigação para analisar os impactos das intervenções nos acervos de autorias dos alunos e na ocorrência de feedbacks.

Os procedimentos metodológicos foram divididos em 3 etapas: (i) aplicação da Arquitetura Pedagógica Y em uma atividade de leitura em uma disciplina de um curso de pós-graduação, formada por uma turma de 15 alunos, (ii) análise da correlação entre as respostas às intervenções e os registros do acervo de certezas e dúvidas dos alunos, (iii) análise da correlação entre autorias e feedbacks.

\section{Referencial Teórico}

As novas possibilidades de comunicação e cooperação, viabilizadas pelas redes de colaboração, rompem as barreiras de tempo e espaço e permitem ao educador o compartilhamento de ideias com seus pares e estudantes, em qualquer tempo e espaço. Os aspectos que permeiam a colaboração podem ser explorados e utilizados como sustentação para ampliar o suporte dessa rede de relações.

Os sistemas colaborativos, também chamados de groupwares, correspondem aos softwares que apoiam as atividades em grupo. Ellis et al. (1991) define groupwares como sistemas baseados em computador que apoiam grupos de pessoas comprometidas em uma tarefa (objetivo) comum e fornecem uma interface para um ambiente compartilhado. Esses sistemas têm como objetivo assistir grupos na comunicação, na coordenação e na colaboração [Ellis et al. 1991].

A taxonomia de Fuks et al. (2007) estende os conceitos tratados por Ellis et al. (1991) e Borghoff e Schlichter (2000), de forma denominar a colaboração como um conceito mais amplo, que por sua vez, integra outros elementos inerentes a qualquer trabalho em grupo. Portanto, a colaboração para Fuks et al. (2007) é a inter- e intra-relação entre comunicação, coordenação e cooperação [Fuks et al., 2008]. De acordo com o exposto, esse artigo se fundamenta no conceito de colaboração de Fuks et al. (2007).

Antes da fase de colaboração ser iniciada, se faz necessária uma fase de planejamento, a articulação. Em um grupo, a distribuição de tarefas entre vários indivíduos demanda um trabalho extra de articulação que envolve o planejamento e a organização destas tarefas, para que sejam realizadas com sucesso. Esta consiste em todas as tarefas envolvidas na preparação do ambiente, agendamento, monitoramento e a coordenação de todas as etapas necessárias para completar as atividades [Schmidt E Bannon, 1992]. Considera-se ainda, como fase do processo de articulação atividades: identificação dos objetivos, o mapeamento destes objetivos em tarefas, a seleção dos participantes e a delegação das tarefas aos participantes. Esta articulação busca garantir que os trabalhos individuais sejam somados e os objetivos comuns alcançados.

Trabalhar cooperativamente demanda esforço adicional para a coordenação de membros. Sem a coordenação é possível que parte dos esforços de comunicação não seja aproveitada na cooperação. Isto é, para que o grupo possa trabalhar em conjunto de forma harmoniosa, é necessário que os compromissos assumidos nas conversações sejam gerenciados para evitar conflitos interpessoais, que possam prejudicá-lo. Desta forma, sem a coordenação, os colaboradores poderão se engajar em atividades conflitantes ou repetitivas [Schmidt e Bannon, 1992].

No trabalho em grupo, a coordenação de atividades é necessária para garantir o cumprimento dos compromissos assumidos na comunicação e a realização do trabalho colaborativo através da soma dos trabalhos individuais. A coordenação organiza o grupo para evitar que esforços de comunicação e de cooperação sejam perdidos e para garantir que as tarefas sejam realizadas da forma mais adequada, no tempo previsto e com os recursos necessários [Raposo e Fuks, 2002].

Quando não é possível pré-articular totalmente as tarefas ou quando é necessário adaptar o planejamento, a coordenação é tratada dinamicamente durante o gerenciamento do andamento das tarefas e 
de suas interdependências. Esta coordenação, definida como "o ato de gerenciar interdependências entre as atividades realizadas para se atingir um objetivo" [Malone e Crowston, 1990], é renegociada de maneira quase contínua ao longo de todo o processo. Apesar de a interdependência ser normalmente positiva - um participante desejando que o trabalho do outro seja bem-sucedido - ela nem sempre é harmoniosa.

Um coordenador pode ser definido para organizar o grupo durante a colaboração, reduzindo a necessidade de pré-articulação. Ele é responsável por organizar os participantes, tarefas e recursos e acompanha a evolução dos processos de trabalho. Alguns grupos operam bem sem a presença de um coordenador explícito: os participantes se organizam dinamicamente enquanto a colaboração ocorre, ajustando-se dinamicamente às mudanças nas tarefas e ao seu entendimento [Dron et al., 2001] apud [Gerosa, 2006]. Esta abordagem é apropriada para grupos pequenos, coesos e com participantes competentes e comprometidos. Muitas vezes é utilizada uma abordagem híbrida, na qual ora parte das tarefas é pré-articulada ( um coordenador é eleito para acompanhar o grupo), ora, em momentos específicos, os próprios participantes se coordenam. O grau de flexibilidade a ser adotado na coordenação depende dos participantes, das tarefas e dos recursos disponíveis. Ao distribuir a coordenação no grupo, reduz-se a dependência do coordenador, liberando-o para tratar da organização do grupo em um alto nível, bem como planejar ações futuras [Durfee, 1988].

Na maioria dos bate-papos e videoconferências, o grupo coordena-se exclusivamente com base na percepção. Nestes casos, a coordenação fica a cargo do protocolo social, caracterizado pela ausência de mecanismos de coordenação explícitos entre as atividades. A coordenação nestas situações é estabelecida culturalmente [Gutwin E Greenberg, 2002]. Por outro lado, atividades cujas tarefas são altamente interdependentes não são satisfatoriamente coordenadas apenas com o protocolo social. Nestes casos, são utilizados mecanismos de coordenação por software. Um mecanismo de coordenação é um dispositivo voltado a dar suporte ao trabalho de articulação [Schmidt e Simone, 1996].

A modelagem do fluxo de atividades e as regras (condições, permissões, sequenciamento...) são elementos de coordenação a serem considerados no projeto do suporte computacional. É possível caracterizar diferentes tipos de interdependências e identificar mecanismos de coordenação para gerenciá-los [Malone e Crowston, 1990]. As interdependências são relacionadas ao tempo ou aos objetos de cooperação (recursos) [Ellis e Wainer, 1994]. No nível temporal a coordenação lida com o sequenciamento das tarefas, enquanto o nível de objetos lida com o compartilhamento e com a concorrência de acesso [Gerosa, 2006].

\subsection{Modelo 3C de Colaboração}

O Modelo 3C de Colaboração [Ellis, 1991], apresentado e estendido por Fuks et al. (2007) reproduzido na Figura 1, oferece uma taxonomia para os elementos constituintes da colaboração. O modelo conceitual descreve a inter- e intra-relação entre a comunicação, a coordenação e a cooperação.

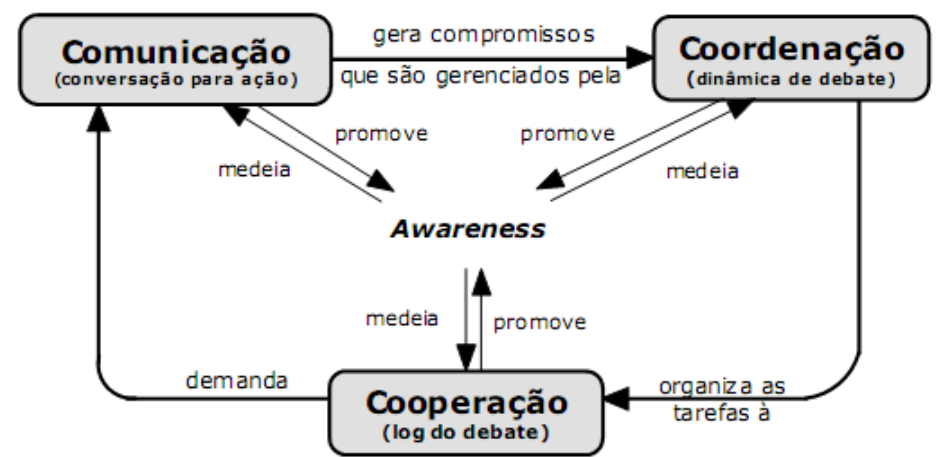

Figura 1. Modelo 3C de Colaboração [Fuks et al., 2007].

O presente trabalho destaca as atividades de coordenação da colaboração, que são responsáveis por 
gerenciar os conflitos, organizar as tarefas com objetivo de prevenir a perda de comunicação e de esforço da cooperação. O trabalho de coordenação pode ser gerenciado por pessoas (protocolo social) e/ou por softwares (sistema de coordenação).

A cooperação é o trabalho conjunto de um grupo de indivíduos sob um espaço compartilhado, objetivando a execução de tarefas. Durante a cooperação acontece a criação e a manipulação de objetos/recursos (artefatos). Ao cooperar, os participantes do grupo se comunicam para renegociar e tomar decisões sobre situações não previstas e, mais uma vez, gerar compromissos, iniciando um novo ciclo.

\subsection{Demandas por coordenação}

Algumas propostas exigem uma complexa coordenação das atividades colaborativas. Dinâmicas que configuram mudanças de papéis de um mesmo participante durante a realização das atividades exige um controle de permissões, a fim de respeitar os compromissos assumidos e evitar problemas no fluxo da dinâmica. Deste modo, as alterações da associação entre participante (usuário) e atividade podem ocorrer em um determinado momento. Os mecanismos de coordenação do groupware devem garantir que as permissões de escrita e leitura durante as atividades ocorram sem falhas. Como características fundantes do groupware, as atividades de coordenação devem garantir que as regras sejam respeitadas em cada perfil de participante, considerando todos os espaços virtuais do domínio, e que o controle das permissões evite perda dos registros individuais e coletivos.

Aprendizagem colaborativa requer um ambiente diferente do tradicional, já que professor e alunos desempenham novos papéis, principalmente quando se ultrapassa a pedagogia transmissiva para uma pedagogia centrada no aluno. As metodologias ativas pressupõem alunos ativos e responsáveis por sua própria aprendizagem. O professor deixa de ser o centro das atenções e o detentor do conhecimento, passando a promover propostas pedagógicas para que os alunos possam progredir por seus próprios esforços. Cabe a ele administrar o trabalho adaptando-o em relação aos temas em estudo, às circunstâncias, ao currículo às áreas específicas e às especificidades dos alunos. Para ambos os papéis, a interação social é imprescindível. Portanto, para atender às demandas de propostas que sustentam estratégias em favor da aprendizagem em rede, os groupwares precisam de sistemas de coordenação mais sofisticados, capaz de reduzir as dificuldades oriundas da colaboração, ao mesmo tempo que facilitar o trabalho do professor (mediador).

\section{Arquiteturas Pedagógicas e a Colaboração}

Diferentes estratégias pedagógicas são apresentadas em Carvalho, Nevado e Menezes (2007), tal como em Castro e Menezes (2012), e se configuram como Arquiteturas Pedagógicas. As Arquiteturas Pedagógicas (APs) são definidas como "suportes estruturantes" para a aprendizagem, que combinam o aparato técnico com a visão pedagógica, numa ideia ecossistêmica (Carvalho; Nevado; Menezes, 2007, p.39). Essa combinação inclui elementos como: abordagem pedagógica, software, internet, Educação a Distância, concepção de interconexão em tempos e espaços múltiplos. A literatura apresenta diversas APs, algumas abordadas resumidamente em Carvalho, Nevado e Menezes (2007) e descritas com mais detalhes em Castro e Menezes (2012).

APs estabelecem uma dinâmica de grupo a ser realizada através de um suporte computacional, com restrições e permissões (regras) bem definidas, protocolos de troca de mensagens que envolvem a autoria e o compartilhamento. O professor deixa de ser o centro das atenções e o detentor do conhecimento, e passa a promover propostas pedagógicas para que os alunos possam progredir por seus próprios esforços [Castro e Menezes, 2012].

A literatura sobre aprendizagem colaborativa apoiada por computador (CSCL) é abrangente e destaca os regulares esforços criados pela comunidade científica em assistir as diferentes práticas pedagógicas. À medida que amplia as possibilidades de novas formas de compartilhamento de 
artefatos em um sistema cooperativo de trocas, o esforço de empregar os mecanismos de coordenação para assistir as diferentes regras de interação se torna mais complexo - principalmente quando combinadas em um mesmo espaço compartilhado. Nesses cenários o suporte computacional pode assumir grande responsabilidade do trabalho de articulação e de coordenação.

\subsection{Arquitetura Pedagógica Leitura Digital}

A Arquitetura Pedagógica para Leitura Digital (APLT) foi projetada a partir de elementos estruturais empregados em outras estratégias pedagógicas, também classificadas como APs: Projeto de Aprendizagem (Fagundes et al., 2006), Debate de Teses (Nevado; Menezes; Vieira Junior, 2011), a Controvérsia Acadêmica (Johnson et al., 1996) e o Júri Simulado (REAL; SILVA, 2007). Para compor a Arquitetura Pedagógica Y buscou-se elementos, os quais demandam dos sujeitos ações (regulações ativas) como posicionamentos, respostas a questões abertas, formalizações de suas compreensões, além de argumentações e manifestações contra-argumentativas.

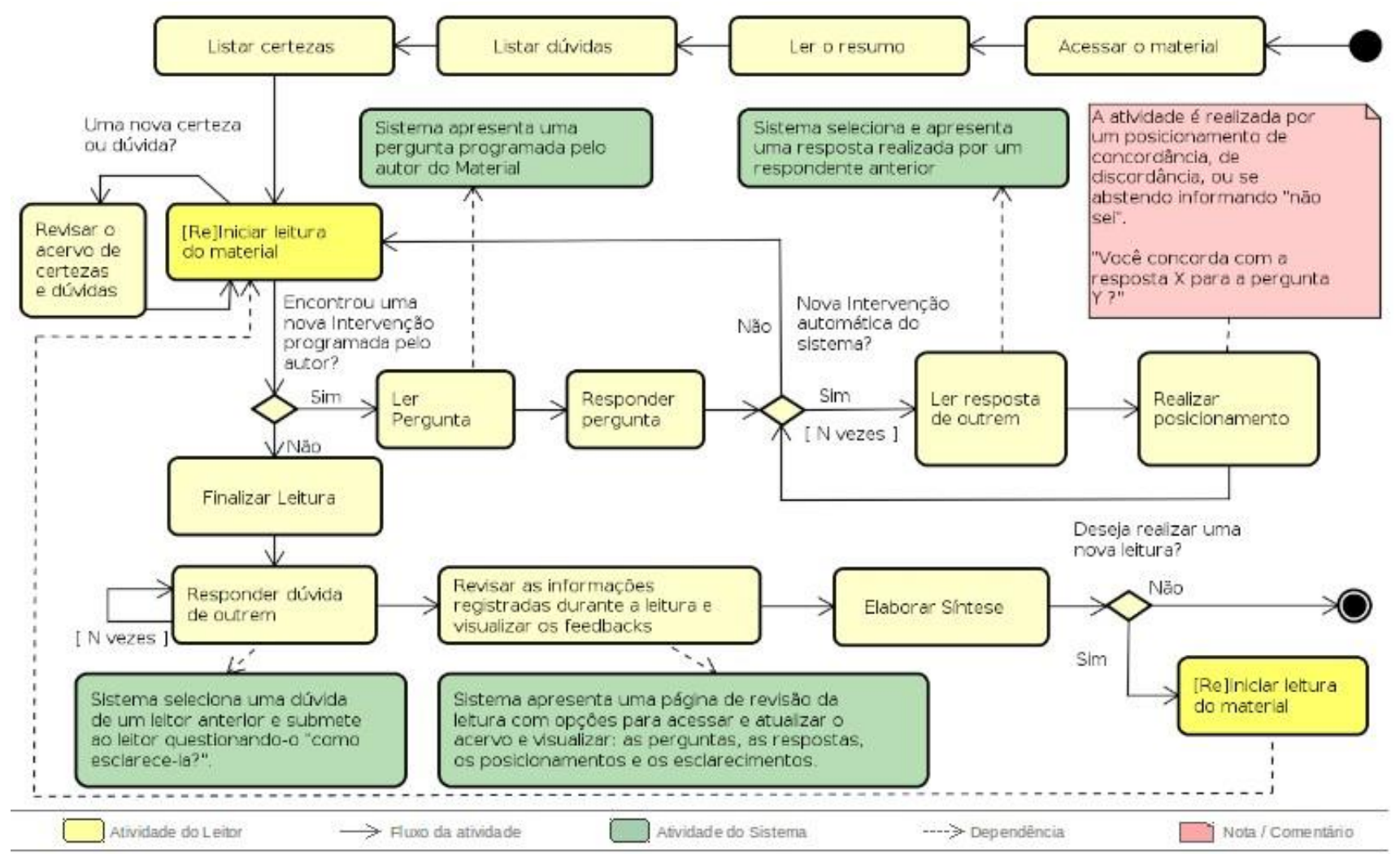

Figura 2. Modelo de fluxo de atividades da APY - adaptado de Vieira Junior e Reategui (2018)

Nesse primeiro grupo de atividades, o leitor é convidado a falar sobre suas dúvidas e certezas a respeito do conteúdo do texto a ser lido. Para melhor assimilar a abrangência do assunto, o leitor é orientado a ler o resumo (preparado pelo autor) acompanhado do título que anuncia a temática do conteúdo do documento. Desta forma, pretende-se, com os resultados das atividades que antecedem a leitura (pré-leitura), produzir um acervo prévio de certezas e dúvidas relacionadas ao conteúdo.

O segundo grupo de atividades é composto pelas atividades que ocorrem durante a leitura. Durante a leitura as "intervenções programadas" serão associadas a um determinado trecho do texto conjunto de caracteres como uma palavra ou um parágrafo do texto. O segmento de texto selecionado será apresentado aos leitores com alteração do formato da fonte (assumindo uma função de hiperlink) para ter destaque visual e simbolizar que não é um link comum. Deste modo, a intervenção na leitura ocorre por uma ação deliberada do próprio leitor por meio do clique do mouse no hiperlink. Acionada a 
intervenção, a pergunta programada pelo autor será apresentada com um espaço para a digitação da resposta. O registro e a visualização das certezas e dúvidas são recursos também disponíveis, durante todo o uso do material, na interface digital. Por fim, após finalizar a leitura, são apresentadas dúvidas a serem esclarecidas, antes de o leitor revisar seu acervo e apresentar uma síntese de suas compreensões.

\subsection{Intervenções}

Para isso, foi projetado e implementado em software o fluxo de atividades (Figura 2), tendo como principal abordagem a intervenção na leitura não invasiva. As intervenções têm por função submeter aos leitores diferentes perguntas. Por um lado, as perguntas visam extrair do sujeito as compreensões sobre seus conhecimentos prévios e sobre a leitura; pelo outro, algumas perguntas auxiliam feedbacks coletivos, ao mesmo tempo que submetem os próprios leitores a se confrontarem com outros pontos de vista.

Em resposta a um conjunto de atividades, o leitor, diretamente ou indiretamente, subsidia algumas intervenções projetadas para outros leitores. Para alcançar este propósito, um conjunto de atividades de intervenções é coordenado pelo suporte computacional para ser realizado antes, durante e após a leitura do texto digital. As atividades de intervenção ocorrem em três eixos de projeção ao leitor: "intervenções fixas", "intervenções programadas" e "intervenções automáticas". As intervenções fixas ocorrem antes e depois da leitura nas atividades e listar certezas/dúvidas e na atividade de elaboração de síntese. As intervenções programadas são previamente elaboradas pelo autor do texto, formatadas em perguntas abertas e configuradas para ocorrer durante a leitura do texto digital - a quantidade de perguntas e a localização delas no texto são critérios do autor (professor) do material digital. As intervenções automáticas são manipuladas pelo sistema do HiperDidático para serem projetadas em diferentes momentos, ocorrendo durante e depois da leitura.

\section{Procedimentos metodológicos}

A preparação para realizar a coleta de dados ocorreu por meio de 3 atividades: (1) disponibilização para acesso ao Groupware HiperDidático, (2) preparação do material digital para a leitura no groupware, (3) definição das orientações para a execução da atividade em grupo.

A disponibilização do HiperDidático ocorreu por meio de um servidor online em um domínio público, tornando seu acesso público por meio do navegador Web. A atividade ocorreu em uma sala (laboratório de informática) com computadores com acesso ao sistema. Todos os alunos ocuparam, individualmente, um computador.

O material digital foi preparado com um texto de autoria do professor da disciplina (trecho de um livro), contando com aproximadamente 2300 palavras (15500 caracteres) e duas ilustrações. A temática do texto contempla discussão e diretrizes sobre a organização e a escrita de textos científicos. Embora nenhum aluno tivesse tido contato com o texto anteriormente, o assunto era "familiar" para todos, tendo em vista a formação acadêmica e as experiências anteriores na escrita desses manuscritos. O professor elaborou 6 perguntas (intervenções programadas) e, utilizando a interface do sistema, elas foram inseridas ao texto em respectivos trechos associados a temática da questão.

As orientações destinadas aos alunos foram: "realizar a leitura completa do texto", "evitar a leitura de outros materiais durante a atividade" e "por meio do clique no link, acionar as perguntas programadas para respondê-las". A quantidade de registros nos acervos, a quantidade de posicionamento e esclarecimentos eram de acordo com o desejo de cada participante.

A turma de alunos foi composta por 15 alunos, dentre os quais todos tinham formação em Mestrado e estavam cursando uma disciplina de um curso de pós-graduação stricto sensu. Todos concordaram com o termo de consentimento esclarecido e preencheram os dados digitalmente. Inicialmente, 15 participantes iniciaram a atividade, mas 2 alunos precisaram encerrar a atividade antes do previsto. Devido às atividades incompletas dos 2 alunos, a pesquisa teve que desconsiderar seus dados e excluí-los dos resultados. 
A realização da atividade teve duração de 1 hora e 30 minutos. A coleta de dados ocorreu diretamente no HiperDidático, o qual foi desenvolvido para coletar dados temporais das projeções das intervenções, bem como armazenar os registros das ações dos usuários no sistema, incluindo os dados de navegação.

A investigação sobre as contribuições das intervenções está associada ao sistema de coordenação do groupware. Avaliação do sistema de coordenação do trabalho colaborativo foi realizada sob duas perspectivas: (1) a primeira analisa todas as ocorrências das intervenções programadas e automáticas respondidas em correspondência com os registros do acervo de certezas e dúvidas dos alunos, (2) a segunda analisa a correspondência entre a produção de autorias (acervo e respostas às perguntas programadas) e a promoção de feedbacks (posicionamentos e esclarecimentos de dúvidas). Para ambas as análises recorreu-se à prova de correlação de Pearson.

\section{Resultados}

Os resultados apresentados pretendem evidenciar indicadores que permitam avaliar o sistema de coordenação de intervenções, tendo em vista as formalizações (respostas, certezas e dúvidas) e os feedbacks (posicionamentos nas respostas e esclarecimentos de dúvidas).

Nessa seção são apresentadas duas análises que mensuram estatisticamente a associação bivariada do grau de relacionamento entre duas variáveis quantitativas. Na primeira, será analisada a relação entre as intervenções e o acervo. Na segunda, será analisado feedbacks e autorias. Diante da análise de correlação de Pearson (r), pretende-se evidenciar a capacidade de promover trocas, em uma lógica de relações interdependentes, entre dois aspectos gerenciados pelo sistema de coordenação.

A Figura 3 apresenta um gráfico de dispersão entre duas variáveis: Intervenções e registros no acervo. Utilizando pontos azuis no plano, as coordenadas $(\mathrm{X}, \mathrm{Y})$ representam cada aluno totalizando 13 pontos. As coordenadas relacionam a quantidade de certezas e dúvidas (eixo Y) e a quantidade de intervenções (programadas e automáticas) respondidas (eixo X).

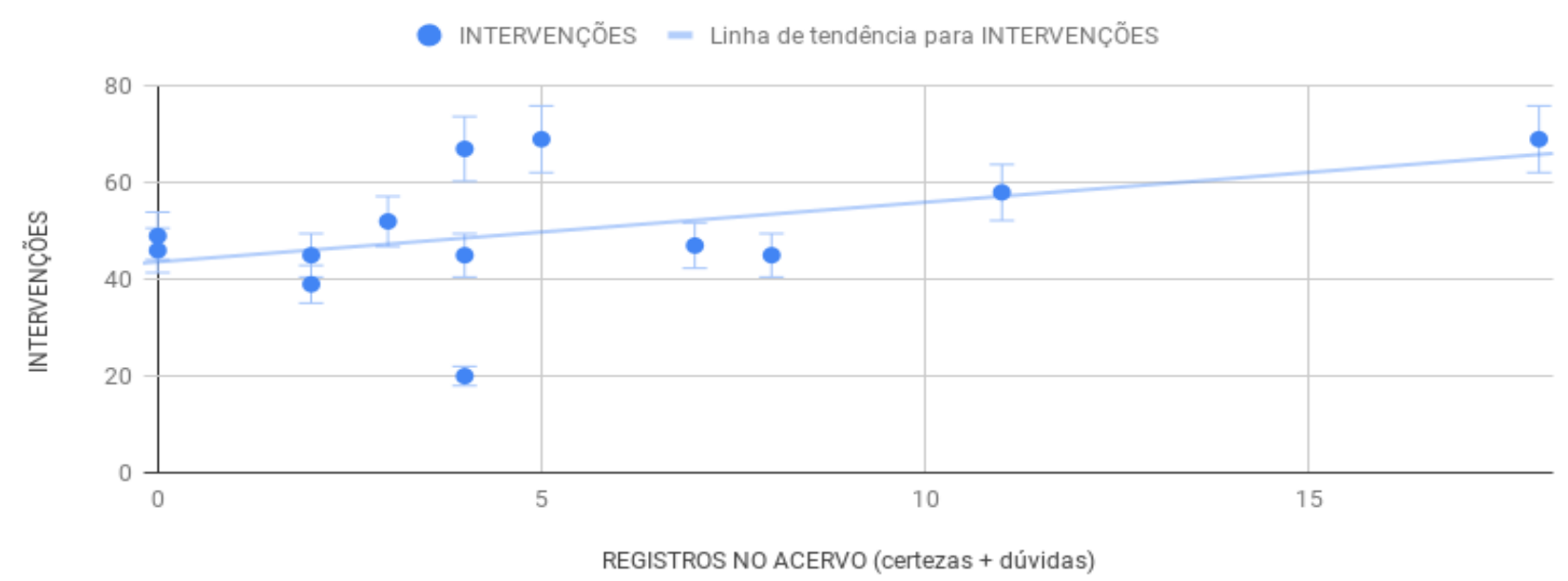

Figura 3. Correlação entre o número de intervenções e as produções no acervo de certezas e dúvidas.

O gráfico apresenta uma covariação positiva e a equação da reta (sinalizada pela linha de tendência) assume um plano que ilustra a relação linear entre as duas variáveis quantitativas. Como pode ser observado em $r=0,44979$, há uma baixa correlação linear positiva entre os dois eixos. Assim, há uma certa influência na reconstrução do acervo, mas as evidências nos mostram que esta relação (covariância) é de baixa significância.

Na Figura 4 é apresentada a progressão dos feedbacks em correspondência interdependente com as produções das autorias ao longo (tempo) da atividade. Nos primeiros minutos a ocorrência 
dos feedbacks supera rapidamente em números frente à ocorrência de criação de autorias. A superação em números é, sobretudo, em função dos posicionamentos. Sendo as autorias os elementos estruturantes para solicitar ofeedback, a interdependência se configura rapidamente.

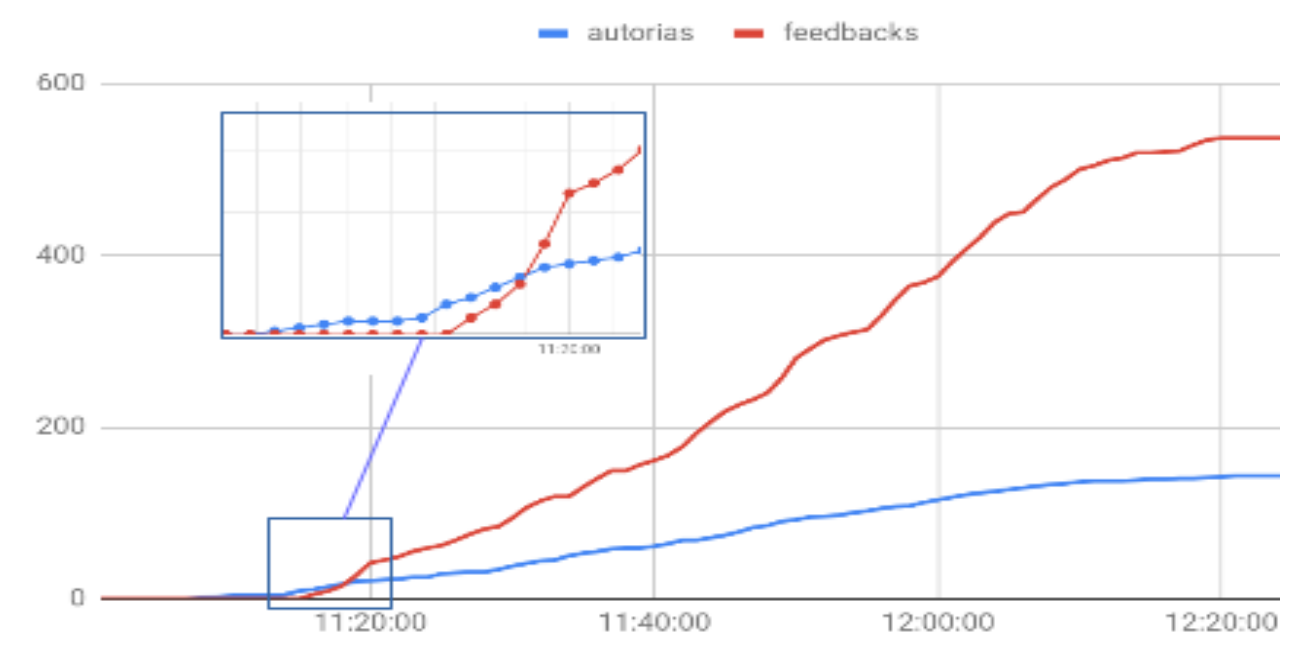

Figura 4. Progressão dos feedbacks em correspondência as produção das autorias

Nessa análise, as autorias consideradas são os registros do acervo e as respostas das perguntas programadas. A análise de correlação de Pearson, entre o número de autorias e a ocorrência de feedbacks, apresentou uma alta correspondência $(\mathrm{r}=0,99044)$ em um nível de significância 'altamente significativo'. As intercorrelações entre as duas variáveis são um resultado direto do emprego dos mecanismos de coordenação no HiperDidático. São os elementos do acervo que subsidiam as intervenções, ocorridas no fim da leitura para, assim, solicitar dos leitores um feedback de esclarecimentos das dúvidas; Por sua vez, as respostas são os elementos que subsidiam as intervenções com questões para posicionamentos.

\section{Conclusões}

Nessa pesquisa foi avaliado o sistema de coordenação de intervenções na leitura, implementado no Groupware HiperDidático e baseado na proposta da APLT. A avaliação foi composta de duas principais análises: análise dos impactos das intervenções sobre o acervo dos leitores; e a correspondência entre as produções das autorias e a ocorrência de feedbacks. A escolha pela utilização de um texto sobre um conteúdo, no qual os alunos tinham muita experiência, pretendeu criar um cenário de avaliação de maior resistência a reconstrução de respostas, dúvidas e certezas. Por um lado, a primeira avaliação pretendia evidenciar os impactos das intervenções, coordenada pelo groupware, nas compreensões sobre o conteúdo, pelo outro identificar a contribuição dos mecanismos de coordenação na promoção de feedbacks em função das autorias elaboradas pelos próprios alunos.

$\mathrm{O}$ resultado da primeira análise evidenciou uma correlação estatística pouco significativa entre as intervenções e a reconstrução do acervo. A interpretação é que há uma certa influência das intervenções sobre as produções de certezas e dúvidas. Ao considerarmos a experiência dos alunos com o conteúdo, a correspondência (baixa correlação linear positiva: $r=0,44979$ ) possui um grau de relevância positiva. No entanto, o resultado nos leva a considerar a influência de outras variáveis, avançando para uma continuidade da investigação sob dois novos eixos: "domínio do conteúdo" tratado pelo texto e análise individual sobre cada intervenção (pergunta).

A segunda análise apresentou uma alta correspondência (alta correlação linear positiva: $r=$ 0,99044) entre a produção de autorias e a ocorrência de feedbacks em um nível de significância 'altamente significativo' durante a atividade com a turma. Os resultados sugerem um sistema de coordenação capaz de ampliar a ocorrência de feedbacks, mesmo com baixa produção de autorias. 
Embora recomendado a responder as perguntas apresentadas pelo sistema, nenhuma pergunta tinha carácter obrigatório, de modo que o leitor poderia ignorar/desistir de responder qualquer pergunta apresentada. Ou seja, o leitor poderia não se submeter às intervenções. No entanto, foi constatado um crescimento de feedbacks em função das autorias ao longo da atividade. A facilidade de recuperar os artefatos (autorias) gerados e realocar em um sistema de intervenção, ao longo do uso do material, permitiu ao groupware HiperDidático dinamizar as atividades de feedback. A avaliação nos evidencia que o sistema de coordenação do trabalho colaborativo teve uma alguma influência na formulação de certezas e dúvidas durante a atividade observada e um papel importante na promoção de feedbacks em uma dinâmica de perguntas e respostas.

\section{Referências}

Durfee, E. H.. Coordination of Distributed Problem Solvers. Kluwer Academic Publishers, Norwell, MA, USA, 1988.

Eliis, C.A.; Wainer, J. A. Conceptual Model of Groupware, In T. Malone (ed)Conference on ComputerSupported Cooperative Work (CSCW). P 79-88, 1994.

Ellis, C. A.; Gibbs, S. J.; Rein, G.. Groupware: Some issues and experiences. Commun. ACM , 34(1). p. 39-58, 1991.

Fagundes, L. C.; Nevado, R. A.;; Basso, M. V.; Bitencourt, J.; Menezes, C. S.; Monteiro, V. C. P. Projetos de aprendizagemuma experiência mediada por ambientes telemáticos. Revista brasileira de Informática na Educação, 14(1), 2006.

Fuks, H.; Raposo, A.; Gerosa, M. A.; Pimentel, M.; Lucena, C. J. P. The 3c collaboration model. In The Encyclopedia of E-Collaboration , p. 637-644, 2007.

Fuks, H., Raposo, A.; Gerosa, M. A.; Pimentel, M.; Filippo, D.; Lucena, C. (2008). Inter-and intrarelationships between communication coordination and cooperation in the scope of the $3 \mathrm{C}$ Collaboration Model. In 12th International Conference on Computer Supported Cooperative Work in Design. Xi'an, China: IEEE Press,. p. 148-153. ISBN 978-1-4244-1650-9.

Gerosa, M. A.; Pimentel, M.; Fuks, H.; de Lucena, C. J. P. Development of groupware based on the 3c collaboration model and component technology. In: Proceedings of the 12th International Conference on Groupware: Design, Implementation, and Use. Berlin, Heidelberg: Springer-Verlag, 2006. (CRIWG'06), p.302-309. ISBN 3-540-39591-1, 978-3-540-39591-1.

Gutwin, C.; Greenberg, S. A Descriptive Framework of Workspace Awareness for Real-Time Groupware, Computer Supported Cooperative Work, Vol. 11, No. 3-4, 2002, p.411-446.

HiperDidático (2019). http://www.hiperdidatico.com.br. Acesso em: 20 julho. 2019.

Johnson, D; Johnson, R.; Smith, K., Academic controversy: enriching college instruction through intellectual conflict. ERIC digest. Graduate School of Education and Human Development, George Washington University, 1996.

Malone, T. W.; Crowston, K. What is coordination theory and how can it help design cooperative work systems? In: Proceedings of the 1990 ACM Conference on Computer-supported Cooperative Work. New York, NY, USA: ACM, 1990. (CSCW' '90), p. 357-370.

Nevado, R. A; Carvalho, M. J. S; Menezes, C. S. (2007). Arquiteturas pedagógicas para educação a distância. In Aprendizagem em rede na educação a distância: estudos e recursos para formação de professores.

Nevado, R. N.; Menezes, C. S.; Vieira Junior, R. R. M. (2011). Debate de teses - uma arquitetura pedagógica. In Anais do Simpósio Brasileiro de Informática na Educação, p. 820-829.

Piaget, J. Abstração Reflexionante. As relações lógico-aritméticas e a ordem das relações espaciais. Tradução: Fernando Becker e Petronilha B. G. da Silva. Porto Alegre: Artes Médicas, 1995.

Schmidt, K.; Bannon, L. Taking CSCW seriously. Computer Supported Cooperative Work (CSCW), v. 1, n. 1, p. 7-40, Março 1992. ISSN 1573-7551.

Schmidt, K.; Simone, C. (1996) Coordination mechanisms: Towards a conceptual foundation of CSCW systems design. Computer Supported Cooperative Work, 5(2-3), 155-200.

Vieira Junior, R. R. M.; Reategui, E. B. Arquitetura Pedagógica para leitura de textos digitais. In: Simpósio Brasileiro de Informática na Educação (SBIE). Fortaleza, 2018, p.876-885. DOI:10.5753/cbie.wcbie.2018.876 